\title{
Letter on the Article: Role of Contrast-Enhanced Ultrasound (CE/US) in Paediatric Practice: An EFSUMB Position Statement
}

\section{Dear Editor,}

I have read with interest the EFSUMB position statement concerning the role of contrast-enhanced ultrasound (CEUS) in pediatrics [1].

The authors claim to be able to reduce the use of CT and MRI considerably. This should be the aim of the article. I doubt that this is possible with CEUS while maintaining an acceptable diagnostic/therapeutic standard, especially with regard to masses.

1. Focal liver lesions: in severe cases, the major role of imaging is to accurately define the extent of a lesion in relation to the hepatic lobar anatomy and vascular and biliary structures for preoperative planning and to monitor tumor response to chemotherapy or radiation (i. e., hepatoblastoma, lymphoma, etc.). CT and MRI are necessary. The article with the largest series does not include malignant lesions, the sensitivity and accuracy are not calculated, the specificity for identifying a benign lesion is $98 \%$ and the negative predictive value is $100 \%$ [2]. Multifocal lesions in newborns/infants are usually hemangiomas and neuroblastoma metastases. Doppler ultrasound usually allows differential diagnosis.

2. Renal lesions that necessitate CT or MRI are usually Wilms tumors and CEUS plays no role in oncologic protocols. Renal cyst classification with the modified US Bosniak system is not a pediatric problem.

3. Lung CEUS may improve the diagnostic accuracy of grayscale US supplemented by color and power Doppler imaging for the differentiation of consolidated lung from cavitating pneumonia in children. In this sense, the gold standard is CT and currently does not change management. Therefore, CEUS is useless.

4. The most common adrenal mass in children is neuroblastoma. Staging is based on CT/MRI, metaiodobenzylguanidine (MIBG), bone scintigraphy. In the neonatal period differential diagnosis with adrenal hemorrhage depends on the follow-up.

5. With respect to the spleen, the authors report the differential diagnoses of splenic lymphoma and hemangioma as a suitable indication in children. Lymphomas in children are systemic diseases. Except for in infants, so-called hemangiomas are vascular malformations that are hypervascularized on color Doppler [3]. Lymphatic malformations usually require a complete detailed anatomic assessment.

6. Regarding inflammatory bowel diseases, the ESGAR/ESPR consensus statement concludes that the routine use of contrast-enhanced US (CEUS) is currently not recommended [4].

7. Although not widely available or clinically used, CEUS (CT as the reference standard) has a sensitivity of $92 \%$ and a specificity of $100 \%$ for liver and spleen injuries and $80-90 \%$ for renal injuries [5].

I believe that the review of the literature is optimistic and inhomogeneous. In particular, it is evident that the conclusions come mainly from radiological experience acquired on the basis of adults.

\section{Author of the letter to the editor}

Paolo Toma

imaging, Ospedale Pediatrico Bambino Gesu, Roma, Italy

Correspondence

Dr. Paolo Toma
imaging, Ospedale Pediatrico Bambino Gesu
piazza S Onofrio 4
00165 Roma
Italy
paolo.toma@opbg.net

\section{References}

[1] Sidhu PS, Cantisani V, Deganello A et al. Role of contrast-enhanced ultrasound (CEUS) in paediatric practice: An EFSUMB position statement. Ultraschall in Med 2017. DOI: $10.1055 / \mathrm{s}-0042-110394$

[2] Jacob J, Deganello A, Sellars ME et al. Contrast Enhanced Ultrasound (CEUS) Characterization of Grey-scale Sonographic Indeterminate Focal Liver Lesions in Paediatric Practice. Ultraschall in Med 2013; 34: 529 540

[3] Taylor SA, Avni F, Cronin CG et al. The first joint ESGAR/ ESPR consensus statement on the technical performance of cross-sectional small bowel and colonic imaging. Eur Radiol 2016. DOI: 10.1007/s00330-016-4615-9

[4] Mulliken JB et al. Young's Vascular Anomalies: Hemangiomas and Malformations. Oxford University Press. 2013

[5] Valentino M, Serra C, Zironi G et al. Blunt Abdominal Trauma: Emergency Contrast-Enhanced Sonography for Detection of Solid Organ Injuries. Am J Roentgenol 2006; 186: $1361-1367$

Bibliography

DOI http://dx.doi.org/10.1055/s-0043-101520 Published online: April 11, 2017 | Ultraschall in Med 2017; 38: 446-449

(c) Georg Thieme Verlag KG Stuttgart · New York ISSN 0172-4614 